plification of $\beta$-globin genomic DNA for diagnosis of sickle cell anemia. Proc. Natl. Acad. Sci. USA 86:2757-2760.

This work is supported by INTAS grant no. 99-01391, INCO-Copernicus grant no. ICA2-CT-2000-10056, and RFBR grant no. 02-04-49890. Address correspondence to Dr. Evgeny Imyanitov, Group of Molecular Diagnostics, N.N. Petrov Institute of Oncology, St.-Petersburg 197758, Russia. e-mail: evgeny@imyanitov.spb.ru

Received 24 May 2002; accepted 2 July 2002.

E.N. Imyanitov, K.G. Buslov, E.N. Suspitsin, E.Sh. Kuligina, E.V. Belogubova, M.Yu. Grigoriev, A.V. Togo, and K.P. Hanson

N.N. Petrov Institute of

Oncology

St.-Petersburg, Russia

For reprints of this or any other article, contact Reprints@BioTechniques.com

\section{Construction of a Broad Host Range Cloning Vector Conferring Triclosan Resistance}

BioTechniques 33:490-492 (September 2002)

Triclosan is an antimicrobial found in deodorants, toothpaste, hand lotions, and acne treatments, along with many other household products and is an effective inhibitor of both Gram-positive and Gram-negative species (8). Recently, there has been increased research into the mechanisms whereby bacteria become resistant to the antimicrobial effects of triclosan. McMurry et al. $(10,11)$ identified the enoyl-acyl carrier protein (ACP) reductase, FabI, as the main intracellular enzyme target of triclosan in E. coli. It has also been shown, for example, that some bacteria contain orthologous enoyl-ACP reductases, namely FabL and FabK, which are not inhibited by triclosan $(6,7)$. Bacteria that contain these alternate enzymes are able to tolerate higher concentrations of triclosan than those bacteria that have only the FabI-type enoyl-ACP reductase $(6,7)$.

The construction of a vector with a triclosan resistance determinant would make triclosan a useful addition to the selection agents that are currently available. Triclosan is effective against a range of bacteria at less than $10 \mathrm{ppm}$ (8). In E. coli, even mutants with increased resistance are still inhibited at approximately $10 \mathrm{ppm}$ triclosan (10, 11). Triclosan is also nontoxic to humans $(3,8)$. These characteristics make triclosan an attractive selective agent. However, no cloning vectors are currently available with triclosan resistance determinants. Here we report the construction of a broad host range plasmid that is amenable to blue-white screening and that contains a triclosan resistance marker.

The enoyl-ACP reductase gene $f a b L$ (6), including the promoter, was amplified by PCR from Bacillus subtilis chromosomal DNA using the primers YgaABam (5'-GGATCCTTAAACGAGCAGTGAGCGTCCGCCGTC-3') (6) and YgaABam 3 (5'-GGATCCCCCTTTAAAGTTCTTGCCA-3'). Both primers were designed with BamHI sites (in bold) on the $5^{\prime}$ ends. PCR product was ligated into pGEM-T $^{\circledR}$ Easy (Promega, Madison, WI, USA) and transformed into One-Shot ${ }^{\mathrm{TM}}$ (Invitrogen, Carlsbad, CA, USA) chemically competent cells following the manufacturers' instructions. Clones containing the $f a b L$ insert were identified through gel electrophoresis of BamHI-digested plasmid. The $f a b L$ fragment was purified from the agarose gel and ligated into $B g l I I-d i g e s t e d$ pBBR1-MCS2 (9). The ligation reaction was electroporated into $E$. coli $\mathrm{S} 17$ cells, and transformants were selected on LB agar containing 50 ppm kanamycin and $10 \mathrm{ppm}$ triclosan. Several transformants were obtained, and three were analyzed by restriction digest with HindIII. All contained fabL in the same orientation. This new vector was called pBBRT (Figure 1).

E. coli $\mathrm{S} 17$ containing pBBRT was streaked on LB containing up to 10000 ppm triclosan to determine the level of resistance. Also, growth curves of $E$. coli S17 with or without pBBRT were determined in liquid LB medium at varied concentrations of triclosan. In a 96well microplate, overnight culture of either S17 or S17 pBBRT was inoculated into LB containing 0-100 ppm triclosan. Absorbance at $600 \mathrm{~nm}$ was measured over time in a MicroQuant ${ }^{\mathrm{TM}}$ (Bio-Tek ${ }^{\circledR}$ Instruments, Winooski, VT, USA) spectrophotometer with $\mathrm{KC} \mathrm{Ju}$ nior $^{\mathrm{TM}}$ software (Bio-Tek Instruments).

E. coli $\mathrm{S} 17 \mathrm{pBBRT}$ was able to grow in liquid media containing up to 25 ppm triclosan. At $50 \mathrm{ppm}$ and higher, growth of S17 pBBRT was slow, although not completely inhibited (data not shown). The parental E. coli S17 strain has a minimum inhibitory concentration of less than $1 \mathrm{ppm}$ (data not shown). On LB agar plates, however, S17 pBBRT was able to grow in the presence of up to $10000 \mathrm{ppm}$ triclosan, whereas the parent strain could not grow on plates containing $2 \mathrm{ppm}$ triclosan. The tolerance of higher triclosan levels by S17 pBBRT on the plate may be due to diffusional constraints of the excess triclosan, as triclosan is only soluble to approximately $10 \mathrm{ppm}$ in water (4). To demonstrate the utility of this construct in other organisms, pBBRT was also transferred 
via filter mating to Ralstonia eutropha JMP134. Transconjugates were capable of growth on $10 \mathrm{ppm}$ triclosan plates, whereas the parental strain was not.

To ensure the usefulness of this construct for cloning, we inserted a GFP gene ( $g f p$ ) into the EcoRV restriction site of the multiple cloning region. The plasmid pJBA103 (1) was digested with NotI to obtain an approximately 2000-bp fragment containing $g f p$. This fragment was gel-purified, end-polished using $P f u$ DNA polymerase (Stratagene, La Jolla, CA, USA), and then blunt-end ligated into EcoRV-digested pBBRT. The ligation reaction was electroporated into E. coli JM109, and transformants were selected by plating onto LB agar containing triclosan $(10 \mathrm{ppm})$. Colonies containing the $g f p$ insert were identified through both blue-white screening and fluorescence of GFP under a UV light source. Two white colonies glowed unambiguously under UV light, confirming that gfp was successfully cloned into pBBRT. The sequence of the cloning vector $\mathrm{pBBRT}$ has been submitted to GenBank $^{\circledR}$ and is available under accession no. AF504908.

In this study, the alternate enoylACP reductase gene, $f a b L$, from $B$. subtilis was successfully inserted into the broad host range plasmid pBBR1MCS2 to create the triclosan resistance cloning vector, pBBRT. A majority of the restriction sites in the multiple

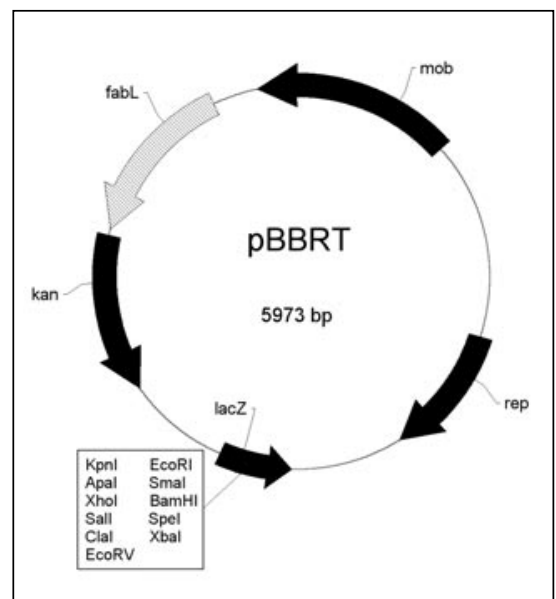

Figure 1. Map of the cloning vector pBBRT. Restriction sites of the multiple cloning region are indicated. The triclosan resistance gene $f a b L$ is represented by the shaded arrow. Restriction sites used for insertion are indicated on either side of the gene. cloning region of the parent plasmid pBBR1-MCS2 remains unique, except for the HincII, SacI, and HindIII sites, as a result of these restriction sites being present in the $f a b L$ gene. The plasmid pBBRT is also a functional cloning vector, as shown by the cloning of $g f p$.

The natural $B$. subtilis promoter contains -10 and -35 regions very similar in spacing and sequence to the $E$. coli housekeeping consensus and should therefore be constitutively expressed in E. coli. It can be assumed that $f a b L$ was indeed expressed in $E$. coli containing pBBRT because of its high level of resistance to triclosan. The normal minimum inhibitory concentration of $E$. coli is less than $1 \mathrm{ppm}(8,11)$, but with pBBRT, E. coli was able to grow uninhibited at $10000 \mathrm{ppm}$ triclosan on solid media. This vector also allowed for the use of triclosan as a selection agent in the strain $R$. eutropha JMP134 (data not shown).

The construction of cloning vectors containing triclosan resistance determinants will expand the array of selection agents available to researchers. This could be of particular use in cases where resistance is already present to traditional antibiotics. Although some bacterial species such as Pseudomonads are resistant to triclosan because of the presence of multidrug efflux pumps (5), triclosan is effective against a wide range of bacterial species (8). Plasmids based on the parent vector, pBBR1MCS, have been widely used because they have a high copy number, a broad host range, and are not members of any known incompatibility groups (2). The combination of these attributes in a single construct should prove useful for routine cloning and expression.

\section{REFERENCES}

1.Anderson, J.B., C. Sternberg, L.K. Poulsen, S.P. Bjorn, M. Givskov, S. Molin. 1998. New unstable variants of green fluorescent protein for studies of transient gene expression in bacteria. Appl. Environ. Microbiol. 64:2240-2246.

2.Antoine, R. and C. Locht. 1992. Isolation and molecular characterization of a novel broadhost-range plasmid from Bordetella bronchiseptica with sequence similarities to plasmids from Gram-positive organisms. Mol. Microbiol. 6:1785-1799.

3.Bhargava, H.N. and P.A. Leonard. 1996. Triclosan: applications and safety. Am. J. Infect. Control 24:209-218.
4.Ciba Specialty Chemicals. General information on Irgasan/Irgacare/Irgacide: Brochure no. 2520. Ciba Specialty Chemicals, Basel, Switzerland.

5.Chuanchuen, R., K. Beinlich, and H.P. Schweizer. 2000. Multidrug efflux pumps and triclosan resistance in Pseudomonas aeruginosa. Abstr. Gen. Meet. Am. Soc. Microbiol. 100:8.

6.Heath, R.J., N. Su, C.K. Murphy, and C.O. Rock. 2000. The enoyl-[acyl carrier protein] reductases, FabI and FabL, from Bacillus subtilis. J. Biol. Chem. 275:40128-40133.

7.Heath, R.J. and C.O. Rock. 2000. A triclosan-resistant bacterial enzyme. Nature 406:145-146.

8.Jones, R.D., H.B. Jampani, J.L. Newman, and A.S. Lee. 2000. Triclosan: a review of effectiveness and safety in health care settings. Am. J. Infect. Control. 28:184-196.

9.Kovach, M.E., P.H. Elzer, D.S. Hill, G.T. Robertson, M.A. Farris, R.M. Roop, II, and K.M. Peterson. 1995. Four new derivatives of the broad-host-range cloning vector pBBR1MCS, carrying different antibiotic-resistance cassettes. Gene 166:175-176.

10.McMurry, L.M., M. Oethinger, and S.B. Levy. 1998. Overexpression of marA, soxS, or acr $A B$ produces resistance to triclosan in laboratory and clinical strains of Escherichia coli. FEMS Microbiol. Lett. 166:305-309.

11.McMurry, L.M., M. Oethinger, and S.B. Levy. 1998. Triclosan targets lipid synthesis. Nature 394:531-532.

Jeanne Kagle is a Howard Hughes Medical Institute Predoctoral Fellow. We would also like to acknowledge M.E. Kovach at Louisiana State University Medical Center for providing pBBRI-MCS2 and Ciba Specialty Chemicals for providing Triclosan (Irgasan DP 300). Address correspondence to Dr. Anthony G. Hay, B53 Wing Hall, Cornell University, Ithaca, NY 14853, USA. e-mail: agh5@cornell.edu

Received 3 December 2001; accepted 2 May 2002.

\section{Jeanne Kagle and Anthony G. Hay Cornell University Ithaca, NY, USA}

For reprints of this or any other article, contact Reprints@BioTechniques.com 